\title{
Globalisierung durch reduzierte Fachwörter, oder Elemente einer beinahe universellen Lingua franca? ${ }^{1}$
}

\author{
Márta MURÁNYI-ZAGYVAI
}

\begin{abstract}
Globalization by means of reduced technical terms, or elements of an almost universal lingua franca?

This paper focuses on multi-segmental acronyms (Kurzwörter), whose use was initially condemned by German linguistic purists as a manifestation of language decay. However, the desire for abbreviation has not abated: multi-segmental acronyms are being used more and more. Without them, technical language would no longer be conceivable today. But are they only popular because of their brevity? This article investigates the advantages of using multi-segmental acronyms in technical texts, as well as exploring a kind of globalization process affecting technical languages, manifested in a new kind of embedding of English and English-transmitted technical terms.
\end{abstract}

Keywords: globalization, technical languages, acronyms, borrowing

DOI: doi.org/10.15452/StudiaGermanistica.2020.27.0003

\section{Einleitung}

Der Titel des Beitrags soll auf die Annahme hinweisen, dass ein relativ junger Teil des Fachwortschatzes, die englischen und englisch transmittierten multisegmentalen Kurzwörter in den Fachterminologien der modernen Sprachen eine besondere Rolle spielen dürften: als spezielle Lehnwörter tragen sie der Globalisierung der Fachsprachen ,unbemerkt" bei, indem sie sich - anhand ihrer außergewöhnlichen Flexibilität - den Zielsprachen sehr leicht und daher sehr schnell anpassen. Diese Anpassung vollzieht sich auf mehreren Ebenen der Sprache. Im Folgenden wird - nach einer kurzen terminologischen Klärung - den Fragen nachgegangen, was für die Entlehnung von multisegmentalen Kurzwörtern charakteristisch ist, welche Vorteile der Gebrauch von entlehnten multisegmentalen Kurzwörtern in Fachtexten bietet, und welchen Wandel ihre Verwendung in den Terminologien der Fachsprachen verursacht. Im Folgenden wird dieser Prozess am Beispiel von Deutsch und Ungarisch als Zielsprachen und Englisch als Herkunftssprache veranschaulicht. Die analysierten Beispiele stammen in erster Linie aus der Fachsprache der chemischen Analytik.

Diese Veröffentlichung ist mit Unterstützung des Projekts EFOP-3.6.1-16-2016-00001 entstanden. 


\section{Kurzwortbildung und Typen des Kurzwortes}

Im vorliegenden Beitrag wird davon ausgegangen, dass Kurzwörter in den letzten Jahrzehnten in den modernen (Fach)sprachen mit Vorliebe verwendet werden, und dass sich die Reduktion (in der deutschsprachigen Fachliteratur Kurzwortbildung genannt) neben Komposition und Derivation als dritte Wortbildungsart angesehen wird (vgl. Murányiné Zagyvai 2017).

Kurzwörter (ung. $v b<$ világbajnokság = Weltmeisterschaft'; dt. Lkw < Lastkraftwagen; engl. $C D<$ compact disc) und Abkürzungen (ung. pl. < például = dt. z. B. < zum Beispiel= engl./lat. e. g. $<$ exemplī grātia $=$ for example) sind nicht identisch. Letztere stellen nur eine graphisch gekürzte Form eines Lexems dar, Kurzwörter werden dagegen sowohl in der schriftlichen als auch in der mündlichen Kommunikation verwendet, sie sind Wortbildungsprodukte.

In Anlehnung an Kobler-Trill (1994) verstehe ich unter Kurzwörtern alle Wortbildungsprodukte, die durch Reduktion eines Basislexems (einer Langform) entstehen. Kurzwörter enthalten also Segmente der Langform (entweder einzelne Buchstaben oder Sequenzen von ihnen, eventuell ganze Silben), sie stellen Dubletten zu ihrem Basislexem dar. Kurzwörter und ihre Langformen sind Synonyme, sie beziehen sich auf das gleiche Denotat.

Zur Typologie der Kurzwörter gibt es verschiedene Ansätze (vgl. Murányi-Zagyvai 2014). Die bekannteste von ihnen ist die von Kobler-Trill. Die Autorin unterscheidet drei Grundtypen der Kurzwörter: unisegmentale, multisegmentale und partielle (Kobler-Trill 1994:20). Unisegmentale Kurzwörter bestehen aus einem zusammenhängenden Teil ihrer Langform, meist aus dem Anfang, z. B. dt. Prof< Professor; multisegmentale Kurzwörter setzen sich aus mehreren Segmenten ihrer Langform zusammen, die aber in der Langform nicht zusammenhängend auftreten, z. B. dt. $L k w<$ Lastkraftwagen. Partielle Kurzwörter werden ebenso aus mehreren nicht zusammenhängenden Segmenten eines Kompositums gebildet, aber das letzte Segment des Basislexems bleibt ungekürzt, z. B. dt. U-Boot $<$ Unterseeboot.

Je nachdem, aus welchen Langformsegmenten das Kurzwort aufgebaut ist, haben die multisegmentalen Kurzwörter, die die größte Gruppe der Kurzwörter bilden, drei Untertypen. Es sind die Initialkurzwörter, z. B. dt. Lkw < Lastkraftwagen, die Silbenkurzwörter, z. B. dt. Kita < Kindertagesstätte und die Mischkurzwörter, z. B. dt. ASta < Allgemeiner Studentenausschuss. In der vorliegenden Arbeit wird für die Elemente der ersten Gruppe die Bezeichnung Buchstabenkurzwort gebraucht, weil nicht immer die ersten Buchstaben der Langformsegmente an der Kurzwortbildung beteiligt sind.

Den Gegenstand der vorliegenden Untersuchung bildet die Gruppe der multisegmentalen Kurzwörter (im Weiteren MKW), da sie in der fachsprachlichen Lexik viel häufiger als die anderen beiden verwendet werden, und in Bezug auf die Entlehnung besondere Eigenschaften haben.

\section{MKW und Entlehnung}

\subsection{Entlehnung}

Unter Entlehnung versteht man den Vorgang und das Ergebnis der Übernahme eines Wortes, eines Ausdrucks oder einer Wortbildungseinheit aus einer Herkunfts-/Gebersprache in eine Ziel-/Nehmersprache (vgl. Bußmann 2002:193; Donalies 2005:16). Die entlehnten sprachlichen Einheiten werden traditionell durch direkte Sprachraumkontakte übernommen, aber auch ohne diese kann Sprachmaterial aus einer Herkunftssprache in verschiedene Zielsprachen gelangen, indem unterschiedliche Vermittler diesem Prozess beitragen können (vgl. Römer/Matzke 2005:42). Die Rolle des Vermittlers spielten und spielen zum Beispiel Fachsprachen (vgl. Schlaefer 2002:70), vor allem die immer aktuelle internationale Wissenschaftssprache. Wissenschaftliche Forschung ist - mit einigen Ausnahmen - ohne internationale Kooperation nicht denkbar, die gemeinsame Arbeitssprache als Gebersprache hat einen starken Einfluss auf die nationalen Wissenschaftssprachen. In unseren Tagen wird der Austauschprozess durch das Internet, die wichtigste Plattform für Kontakte - sowohl für fachliche als auch für sprachliche - wesentlich beschleunigt. Ein weiterer beschleunigender 
Faktor ist der internationale Handel, der für die neuesten Produkte in möglichst vielen Ländern wirbt und neue Märkte sucht. So kommen Kurzwortbezeichnungen vermarktbarer Innovationen schnell in die Verkehrssprache.

\subsection{Das Korpus und die Befunde}

Die analysierten Daten in der vorliegenden Arbeit stammen aus einem fachsprachlichen Korpus, das aus zehn deutschen und zehn ungarischen Dissertationen und zwei Universitätslehrbüchern zur chemischen Analytik (insgesamt 4013 Seiten) zusammengestellt wurde.

In den Korpustexten waren insgesamt 762 (385 deutsche und 377 ungarische) MKW zu finden. Es muss hier erwähnt werden, dass es relativ viele Fälle gab, in denen nicht eindeutig entschieden werden konnte, ob ein MKW oder eine Abkürzung vorlag, denn zur Untersuchung standen nur schriftliche Texte zur Verfügung, und die Autoren gaben nur eine gemeinsame Liste der verwendeten Abkürzungen, Kurzwörter und Symbole an, aber zur Unterscheidung muss man wissen, ob die betroffene sprachliche Einheit auch in der mündlichen Kommunikation verwendet wird oder nicht. In solchen Fällen wurden Fachleute befragt.

Die überwiegende Mehrheit der Korpusbelege zur chemischen Analytik, insgesamt $89 \%$ der MKW - $86 \%$ der deutschen und $92 \%$ der ungarischen MKW - stellen englische oder englisch transmittierte MKW dar. Unter englisch transmittierten MKW sind Entlehnungen aus dem Englischen zu verstehen, für die charakteristisch ist, dass mindestens ein Buchstabe der MKW aus einem solchen Segment der Langform stammt, das auf eine griechische oder lateinische Wurzel zurückgeführt werden kann. Auch bei der Entscheidung, ob einige ältere MKW englischer oder deutscher Herkunft sind, gab es Schwierigkeiten, denn es war nicht immer eindeutig, welche Sprache die Herkunftssprache und welche die Zielsprache war.

„Rein“ deutsche bzw. ungarische MKW sind zu $12 \%$ und 7 \% vertreten. Die Gruppe der einheimischen MKW besteht aus Bezeichnungen von deutschen und ungarischen Behörden und Institutionen, es gibt nur wenige Gattungswörter (ungarische nur vereinzelt). Einige von diesen sind alte Kurzwörter aus den vierziger oder fünfziger Jahren des 20. Jahrhunderts, als es noch viel weniger MKW gab, Englisch als Wissenschaftssprache noch nicht so dominant war, und als man noch mehr darauf achtete, zu fremden MKW auch einheimische zu bilden, z. B. dt. DC $<$ Dünnschichtchromatographie $=$ ung. VRK $<$ vékonyréteg-kromatográfia $=$ engl. TLC $<$ thin layer chromatography. Es gibt auch jüngere, die als Äquivalente zu englischen MKW gebildet wurden. Ein interessantes Beispiel dafür ist das MKW dt./ung. RFA < Röntgenfluoreszenzanalyse/röntgenfluoreszcencia-analízis $=$ engl. $X R F A<X$-ray fluorescence analysis, bei dem eigentlich nur der erste ,unnötige“ oder vielleicht auch „störende“ Buchstabe des englischen MKW weggelassen wurde. Bei diesem Beispiel spielt natürlich auch eine Rolle, dass die Grundlage der Röntgenfluoreszenzanalyse eine weltweit bekannte deutsche Entdeckung ist, deren deutsche Bezeichnung schon längst vor der Entstehung dieses analytischen Verfahrens existierte - auch im Ungarischen.

Weitere Beispiele aus dem Bereich der Gattungswörter:

dt. FAM $<$ Fertigarzneimittel;

dt. $M A K<$ maximale Arbeitsplatzkonzentration;

dt. $M G V<$ Molekulargewichtsverteilung;

dt. HKA $<$ Hauptkomponentenanalyse;

dt. RPS < Röntgenphotoelektronenspektroskopie/-spektroskop;

dt. $R S A<$ relative Standardabweichung;

dt. $S C<$ Säulenchromatographie;

ung. $L T E<$ lokális termikus egyensúly $=\mathrm{dt}$. LTG $<$ lokales thermisches Gleichgewicht;

ung. $T M B<$ teljes mérési bizonytalanság $=$, vollständige Messunsicherheit‘. 
Beispiele aus dem Bereich der Eigennamen:

dt. $A G S<$ Ausschuss für Gefahrstoffe;

dt. BAM $<$ Bundesanstalt für Materialforschung und -prüfung;

dt. BfR $<$ Bundesinstitut für Risikobewertung;

dt. $B W B<$ Berliner Wasserbetriebe;

dt. DIN $<$ Deutsches Institut für Normung;

dt. $L \ddot{O} G D<$ Landesinstitut für den öffentlichen Gesundheitsdienst,

dt. $M P I<$ Max-Planck-Institut;

ung. KFKI < Központi Fizikai Kutatóintézet = ,Zentrales Forschungsinstitut für Physik';

ung. MTA < Magyar Tudományos Akadémia = ,Ungarische Akademie der Wissenschaften“;

ung. $O B I<$ Országos Borminösitö Intézet $\approx$,Landesinstitut für Weinprüfung';

ung. $O M H<$ Országos Mérésügyi Hivatal $\approx$,Landesinstitut für Messwesen“;

ung. OMMI < Országos Mezögazdasági Minösitö Intézet $\approx$,Landesinstitut für Prüfung von Agrar-

produkten".

\subsection{Besonderheiten der MKW-Entlehnung in den Fachsprachen}

Entlehnungsprozesse sind in den letzten Jahrzehnten viel schneller geworden, Tag für Tag treffen wir neues, fremdes Sprachgut, darunter auch viele MKW. Eine Besonderheit der MKW-Entlehnung besteht darin, dass MKW, in erster Linie Buchstabenkurzwörter viel leichter und daher auch schneller übernommen werden können, und sich schneller anpassen können als nicht reduzierte Lexeme. Die Ursache ihrer hochgradigen Flexibilität liegt darin, dass MKW während der Reduktion ihre morphologische Struktur verloren haben, da sie aus ihrer Langform - mit einigen Ausnahmen nicht bedeutungshaltige Elemente, sondern nur Einzelbuchstaben, einzelne Silben oder silbenähnliche Segmente aufbewahrt haben.

In der vorliegenden Untersuchung werden fachsprachliche MKW, also wohldefinierte Termini berücksichtigt, man kann folglich davon ausgehen, dass eine vollkommene semantische und pragmatische Deckung zwischen herkunfts- und zielsprachigen MKW vorliegt, so wird auf diese Fragen nicht näher eingegangen, und nur die graphematische, phonetische und morphologische Assimilation von fachsprachlichen MKW wird untersucht.

\subsubsection{Graphematische Assimilation: Schreibweise und Schreibung von entlehnten MKW}

In der vorliegenden Arbeit werden Schreibweise und Schreibung nicht synonym verwendet. Unter Schreibweise (als deskriptivem Begriff) wird die Art und Weise verstanden, wie ein MKW in der geschriebenen Sprache von den Sprachbenutzern bewusst gebraucht wird. Die Rechtschreibung (als präskriptiver Begriff) ist dagegen die amtlich festgelegte Schreibweise. Die beiden können identisch sein, müssen aber nicht. So kann es z. B. zu einem Buchstabenwort mehrere Schreibweisen, also Schreibvarianten geben.

Die Rechtschreibregeln der Zielsprachen (in unserem Fall Ungarisch und Deutsch), die sich auf die entlehnten MKW beziehen, sind nicht eindeutig. Selbst in der Schreibung der einheimischen MKW herrschen oft Unsicherheit und Unstimmigkeit, es gibt viele Schwankungen.

In der ungarischen amtlichen Rechtschreibung ist zum Beispiel von Bedeutung, ob das betroffene MKW ein Gattungsname oder ein Eigenname ist. Sowohl für ungarische als auch für fremde Eigennamen-Buchstabenwörter gilt die Regel, dass jeder Buchstabe großgeschrieben wird, unabhängig davon, ob er in der Langform ein Klein- oder ein Großbuchstabe war, z. B. ung. MTA $<$ Magyar Tudományos Akadémia = ,Ungarische Akademie der Wissenschaften'; MÁV < Magyar Államvasutak = ,Ungarische Eisenbahn“ (Laczkó/Mártonfi 2006:391). Die Großschreibung betrifft in solchen Graphemverbindungen auch Grapheme, die zusammen einen Laut bezeichnen, z. B. ENSZ (und nicht ENSz) < Egyesült Nemzetek Szervezete = ,Vereinte Nationen'). Gattungsnamen- 
-Buchstabenwörter werden im Ungarischen eindeutig kleingeschrieben, z. B. áfa <általános forgal$m i$ adó $=$,Mehrwertsteuer'. Eine jüngere Steuerform für Kleinunternehmer heißt aber KATA (und nicht kata) < kisadózó vállalkozások tételes adója. Auch áfa findet man in den ungarischen Texten sehr häufig als $A F A$. Für Silbenkurzwörter sind zum Teil andere Regeln gültig: Eigennamen (auch fremde) sollen mit großem Anfangsbuchstaben geschrieben werden, z. B. Mabisz < Magyar Biztositók Szövetsége = ,Bund Ungarischer Versicherungen', Infoterm < Internationales Informationszentrum für Terminologie, Gattungsnamen dagegen (auch fremde) schreibt man in Kleinbuchstaben, z. B. gyed $<$ gyermekgondozási dij $\approx$,Kindergeld, das die Mutter bis zum zweiten Geburtstag des Kindes bekommt', telex < teletyp exchange (Laczkó/Mártonfi 2006:398).

Zusammenfassend kann also festgestellt werden, dass die ungarische Rechtschreibung im Bereich der MKW sehr kompliziert ist: Selbst wenn man die Regeln kennt, weiß man nicht Bescheid, denn man muss klar sehen, ob ein Gattungsname oder ein Eigenname vorliegt, und was noch problematischer ist: man braucht solide Hintergrundkenntnisse über die Typen der MKW. Mit diesen Regeln sind die meisten Ungarn überfordert. Aber bei den fachsprachlichen MKW lässt die Rechtschreibung eine kleine Tür für die Flucht offen: in den Fachsprachen sei es ,gewohnt", mehrwortige Gattungsnamen durch Buchstabenkurzwörter zu ersetzen, die nur aus Großbuchstaben bestehen (Laczkó/Mártonfi 2006:399), z. B. CB < citizen band; DNS< dezoxiribonukleinsav; EKG< elektrokardiogram; $P V C<$ poli(vinil-klorid), URH $<$ ultrarövidhullám. Damit müsste der Sprachbenutzer einen weiteren Umstand berücksichtigen, wenn er die Rechtschreibregeln einhalten möchte: liegt ein fachsprachliches MKW vor oder nicht? Wo sind die Grenzen zwischen Fachsprachen und Allgemeinsprache? Die Ungarn lösen das Problem auf ihre Art, sie folgen einem einfachen (fremden) Muster: MKW werden im Allgemeinen großgeschrieben, auch in der Allgemeinsprache.

Im Deutschen gibt es für die Rechtschreibung von MKW keine verbindlichen Regeln, nur Vorzugsschreibungen (vgl. Barz 2016:747). Bei MKW, die aus Segmenten von syntaktischen Fügungen gebildet wurden, richtet sich die Groß- bzw. Kleinschreibung im Allgemeinen nach den Segmenten der Langform, z. B. GmbH < Gesellschaft mit beschränkter Haftung. In MKW aus Komposita sind meist alle Segmente großzuschreiben, z. B. $U B<$ Universitätsbibliothek. Und was ist die Praxis? In der Allgemeinsprache werden phonetisch gebunden ausgesprochene MKW wie gewöhnliche Lexeme geschrieben, z. B. dt. Aids $\leq^{2}$ Acquired Immune Deficiency Syndrome. Es gibt allerdings viele Schwankungen, vor allem bei Eigennamen, z. B. dpa < Deutsche Presse-Agentur. Die deutschen Korpusbelege zeugen davon, dass für fachsprachliche MKW - wie im Ungarischen - grundsätzlich die Großschreibung charakteristisch ist, unabhängig davon, was die Langform war.

Sowohl in den deutschen als auch in den ungarisch Korpustexten gab es einige wenige MKW, deren Segmente kleingeschrieben wurden, diese waren aber alle Maßeinheiten, z. B. ppm< parts per million, und die Schreibung der Maßeinheiten richtet sich nach dem Internationalen Einheitensystem, dem SI ( $<$ Système international d'unités). Andere MKW, die nicht großgeschrieben wurden, waren entweder Eigennamen, z. B. dt. BfR < Bundesinstitut für Risikobewertung, oder solche, deren spezielle Schreibung auf andere Ursachen zurückgeführt werden kann. So ist $p H<$ pondus Hidrogenii $=$ ,Wasserstoffionen-Aktivität' eines der ältesten MKW in der Chemie und hat die traditionelle Schreibung aufbewahrt, während in anderen MKW der erste Buchstabe/die ersten Buchstaben den Typ des MKW-Begriffs bezeichnet/bezeichnen, z. B. $m R N A<$ messenger $R N A=$, Boten-RNA'; snRNA $<$ small nuclear ribonucleic acid $=$,kleine nukleäre Ribonukleinsäure'; snoRNA $<$ small nucleolar ribonucleic acid = ,kleine nukleoläre Ribonukleinsäure'; $r R N A<$ ribosomal ribonucleic acid =, ribosomale Ribonukleinsäure“ oder $t R N A<$ transfer ribonucleic acid = ,Transfer-Ribonukleinsäure‘. Einige MKW haben noch - trotz der Terminologisierung - mehrere Schreibungsvarianten, z. B. ToF und $T O F<$ time of flight $=$,Flugzeit" oder $A d S V$ und $A S V<$ adsorptive stripping voltammetry $=$ ,Adsorptions-Stripping Voltammetrie‘. Bei den letzteren ist zu erwarten, dass die gemischte Schreibung allmählich verschwindet.

Das Symbol $\leq$ bedeutet, dass das MKW in der angegebenen Sprache entlehnt worden ist, und aus der angeführten Langform gebildet wurde. 


\subsubsection{Phonetische Assimilation von entlehnten MKW}

Die Aussprache der MKW ist noch weniger geregelt als das Schriftbild, und in dieser Sicht gibt es keinen Unterschied zwischen den einheimischen und den entlehnten MKW. Wenn es im MKW sowohl Vokale als auch Konsonanten bezeichnende Buchstaben vorhanden sind, und wenn die daraus resultierende Lautkombination in der gegebenen Sprache nicht schwierig auszusprechen ist, bestehen grundsätzlich zwei Möglichkeiten, zwischen denen frei zu wählen ist: entweder werden sie phonetisch gebunden ausgesprochen, also wie ein alltägliches einheimisches Wort, z. B. ung. $M A ́ V<$ Magyar Államvasutak = ,Ungarische Eisenbahn'; dt. TÜV $<$ Technischer Überwachungsverein (bei den Silbenkurzwörtern und den Mischkurzwörtern gibt es eigentlich nur diese Möglichkeit), oder phonetisch ungebunden, also mit den Buchstabennamen, z. B. ung. szja < személyi jövedelemadó $=$,Einkommenssteuer'; dt. ADAC<Allgemeiner Deutscher Automobil-Club. Bei entlehnten MKW kommt noch eine Aussprachemöglichkeit hinzu: sie können auch wie in der Herkunftssprache ausgesprochen werden. Welche von den Möglichkeiten realisiert wird, hängt von mehreren Faktoren ab, z. B. davon, wie groß die phonetischen Unterschiede zwischen der Herkunfts- und Zielsprache sind, wie „gern“ die Sprachbenutzer Fremdwörter nach den einheimischen Mustern aussprechen, seit wann das Lehnwort in der Zielsprache benutzt wird, aber auch davon, ob der Sprachbenutzer die Herkunftssprache beherrscht oder nicht. Mischungen der beiden Systeme (z. B. fremde Artikulation mit einheimischer Betonung) sind auch oft anzutreffen. Die ausgesprochenen entlehnten MKW können im Extremfall so große Unterschiede in der Herkunftssprache und in den verschiedenen Zielsprachen aufweisen, dass sie eine völlig andere Lautstruktur haben und ohne Kontext gar nicht identifiziert werden können.

Die Aussprache von MKW wird auch in den Fachsprachen nicht geregelt, obwohl die Standardisierung der mündlichen Form - wenn es um Termine geht - ebenso ein Teil der Terminusbildung sein sollte wie die des Schriftbildes oder die Festlegung des Begriffsinhalts.

Was ist die Praxis? Es gibt zwar keine eindeutigen Regeln, aber einige Tendenzen lassen sich sowohl im Deutschen als auch im Ungarischen beobachten, auch wenn eingehende Untersuchungen noch nötig sind.

MKW, die aus zwei Buchstaben bestehen, werden sowohl im Deutschen als auch im Ungarischen immer phonetisch ungebunden ausgesprochen, aber im Ungarischen liegt der Akzent immer auf der ersten Silbe des Wortes, im Deutschen dagegen meist auf der letzten (hier also auf der zweiten). Zum Beispiel:

- dt. DC < Dünnschichtchromatographie;

- dt. EN < Europäische Norm;

- dt. GC $\leq$ Gaschromatographie;

- ung. GC $\leq$ Gaschromatographie;

- dt. $p H \leq$ pondus Hidrogenii;

- ung. $p H \leq$ pondus Hidrogenii;

- ung. IC $\leq$ ionkromatográfia;

- ung. $P C \leq$ személyi számítógép;

- dt. UV $\leq$ ultraviolett;

- ung. UV $\leq$ ultraviola, ultraibolya.

MKW, die aus drei Buchstaben bestehen, werden im Deutschen im Allgemeinen phonetisch ungebunden ausgesprochen, im Ungarischen dagegen eher phonetisch gebunden. Das ist aber - wie schon erwähnt - keine Regel, es gibt zahlreiche Abweichungen.

- dt. FIA (phonetisch ungebunden) $\leq$ Fließinjektionsanalyse;

- ung. FIA (phonetisch gebunden) $\leq$ folyamatos injektálásos analizis;

- dt. FID (phonetisch ungebunden) $\leq$ Flammenionisationsdetektor;

- ung. FID (phonetisch gebunden) $\leq$ lángionozációs detektor;

- dt. LED (phonetisch ungebunden) $\leq$ Leuchtdiode; 
- ung. $L E D$ (phonetisch gebunden) $\leq$ világító dióda;

- dt. HIV (phonetisch ungebunden) $\leq$ humane Immundefizienz-Virus;

- ung. HIV (phonetisch gebunden) $\leq$ humán immundeficiencia-vírus;

- dt. DIN (phonetisch gebunden) < Deutsches Institut für Normung;

- dt. PET (phonetisch gebunden) $\leq$ Polyethylenterephthalat;

- ung. PET (phonetisch gebunden) $\leq$ polietilén-tereftalát.

Wenn das MKW aus vier oder noch mehr Buchstaben besteht und die oben beschriebenen Bedingungen erfüllt werden, wird sowohl im Deutschen als auch im Ungarischen meist die phonetisch gebundene, an die Zielsprache angepasste Aussprache gewählt, besonders, wenn das MKW mit einem schon bestehenden, keine unangenehme Konnotation aufweisenden Lexem homonym (homophon) ist. Beispiele für MKW mit phonetisch gebundener Aussprache:

- dt. ALAS $\leq$ Archer, Lethrop und Sidhu (Bezeichnung einer Software-Steuerung);

- ung. ANOVA $\leq$ varianciaanalízis (= dt. VA < Varianzanalyse);

- dt. ASCII $\leq$ Amerikanischer Standard-Code für den Informationsaustausch;

- ung. ASCII $\leq$ szabványos amerikai kód információcserére;

- dt. cAMP $\leq$ zyklisches Adenosinmonophosphat;

- ung. cAMP $\leq$ ciklikus adenozin-monofoszfát;

- dt. EDTA $\leq$ Ethylendiamintetraessigsäure, Ethylendiamintetraacetat;

- ung. EDTA $\leq$ etilén-diamin-tetraecetsav, etilén-diamin-tetraacetát;

- dt. GABA $\leq \gamma$-Aminobuttersäure;

- ung. $G A B A \leq \gamma$-aminovajsav;

- dt. MALDI $\leq$ matrix-unterstützte Laser-Desorption/Ionisation;

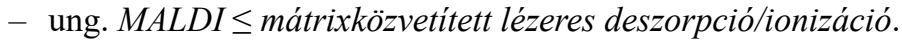

Beispiele für MKW mit Homonymie/Homophonie:

- dt. ELISA $\leq$ enzyme-linked immuno sorbent assay = , heterogener Enzymimmuntest“;

- dt. GRAAS $\leq$ Graphitrohr-AAS (AAS $\leq$ Atomabsorptions-Spektrometrie);

- dt. LAWA < Länderarbeitsgemeinschaft Wasser.

Vereinzelt kommt auch die Kombination fonetisch gebundener und ungebundener Aussprache vor, z. B. bei ung. $N A D H \leq$ nikotinamid-adenin-dinukleotid $+H$, in dem $N A D$ phonetisch gebunden ausgesprochen wird, und $H$ nur phonetisch ungebunden ausgesprochen werden kann.

\subsubsection{Morphologische Assimilation von entlehnten MKW}

Unter morphologischer Assimilation wird in der vorliegenden Arbeit die Anpassung der MKW dem grammatischen System der Zielsprache verstanden.

Im Deutschen bedeutet dies - da die MKW Substantive sind - einerseits die Genuszuweisung, andererseits die Bildung von Genitiv- und Pluralform der Maskulina und Neutra. Die Genuszuweisung ist bei deutschen MKW problemlos, denn - mit ganz wenigen Ausnahmen - hängt das grammatische Geschlecht vom Grundwort im MKW ab, man muss also die Langform kennen. Bei entlehnten MKW ist aber nicht selten, dass die Sprachbenutzer weder die Langform noch eine entsprechendes deutsches Äquivalent kennen (vgl. Barz 2016:747). In den Fachsprachen bezeichnen MKW wohldefinierte Termini, deren Kenntnis vorausgesetzt werden kann, so ist den Fachleuten mindestens die Langform bekannt, auch wenn kein zielsprachiges Äquivalent vorliegt. Im Allgemeinen werden aber MKW ohne Artikel gebraucht (ähnlich wie Eigennamen), so dass der Benutzer keine Probleme mit der Genuszuweisung hat. Im äußersten Falle besteht noch die Möglichkeit, mit dem betroffenen MKW ein Kompositum zu bilden, z. B. statt MALDI kann man MALDI-Verfahren benutzen.

Die Deklination der MKW bereitet keine Schwierigkeiten, wenn man die Regel kennt, dass das -s im Genitiv von Maskulina und Neutra fehlen kann, z. B. der Lkw, des $L k w(s)$. Genauso kann man das -s im Plural weglassen, z. B. der $L k w$, die $L k w(s)$. Ausnahmen sind MKW, die auf einen 
Vollvokal auslauten, und bei denen das -s im Plural nicht fehlen darf, z. B. das UFO, die UFOs (vgl. Barz 2016:748).

Im Ungarischen unterscheidet man keine grammatischen Geschlechter, die Wahl des bestimmten Artikels hängt von der Aussprache ab, genauer davon, ob das ausgesprochene MKW mit einem Vokal oder mit einem Konsonanten beginnt. Es ist also manchmal von Belang, ob die ungarische oder die englische Aussprache gewählt wird, z. B. bei USN < ultrasonic nebulizer =, Ultraschallzerstäuber'.

Bei der Rechtschreibung der affigierten Formen (mit oder ohne Bindestrich, mit oder ohne Signalisierung der Vokaldehnung bei auf einen Vokal auslautenden MKW) müssten sowohl die Aussprache als auch die Schreibung berücksichtigt werden. Bedenkt man aber, mit welchen Schwierigkeiten die Schreibung der MKW verbunden ist (s. 3.3.1), so wundert es nicht, dass die Sprachbenutzer eher die Großschreibung wählen, denn diese Schreibweise sichert, dass das MKW bei der Affigierung in der Schrift unverändert bleiben darf: die Affixe sollen einfach mit Bindestrich an das MKW angehängt werden. Damit ist aber das Problem nur zur Hälfte gelöst, denn auch die Wahl des Affixes wird von der Aussprache (von den Vokalen im MKW) bestimmt, im Ungarischen gibt es nämlich nicht einzelne Affixe, sondern Affixpaare, z. B. -ba/-be = ,in + Akk.' (manchmal sogar drei Formen, z. B. -hoz/-hez/-höz=,zu + Dat.').

Was ist die Praxis? Am häufigsten wird die ungarische Aussprache gewählt, auch wenn die phonetische Assimilation nicht immer gleich erfolgt. Aufgrund der oben erwähnten Regel der Affixwahl kann man im Ungarischen aus der Verwendung der Affixe darauf folgern, welche Aussprache der Autor des Textes in der mündlichen Kommunikation benutzen würde. Zum Beispiel zeigen die Formen BAA-ban, DMMA-val, PGAA-ban, IBMK-ban, TOF-ban, TBA-val davon, dass hier die ungarischen Buchstabennamen gemeint sind.

\section{Vorteile und Folgen des Gebrauchs von entlehnten MKW}

Kurzwörter, vor allem MKW wurden (und werden immer noch) wegen ihrer Benutzerunfreundlichkeit und ihrer „sprachverderbenden Wirkung“ viel kritisiert (vgl. Steinhauer 2000:42), aber die Praxis hat schon längst bewiesen, dass ihr Gebrauch viel mehr Vorteile als Nachteile hat, und dass sie (die Untertypen jeweils andere) wichtige Funktionen in der Kommunikation haben. Im Folgenden werden die positiven Merkmale der MKW erörtert, die anderen Typen der Kurzwörter werden grundsätzlich nicht behandelt.

(1) Von den positiven Eigenschaften wird immer die Kürze, also die Ökonomie als erste erwähnt. Die Kürze ist ein gemeinsamer Vorteil aller Typen der Kurzwörter sowohl in der Allgemeinsprache als auch in den Fachsprachen. Auf diese Kürze kann auch der wichtigste Nachteil, ihre (teilweise) Unmotiviertheit zurückgeführt werden, die von den Kurzworttypen hauptsächlich die MKW betrifft. Bei entlehnten MKW kann - wenn die entsprechenden Fremdsprachenkenntnisse fehlen - mit dieser Unmotiviertheit auch oft verbunden sein, dass die Bedeutung des MKW auch dadurch nicht enträtselt werden kann, dass man die Langform sieht oder hört. Die Unmotiviertheit hat allerdings auch ihre guten Seiten (s. Vorteil 3, 4 und 5).

(2) Die zweite positive Eigenschaft ist Genauigkeit, eine „Vollständigkeit in der Unvollständigkeit"“, d. h. mit der reduzierten Form wird auf den vollen Begriff in seiner Vollständigkeit Bezug genommen, was zum Teil dadurch zum Ausdruck kommt, dass im MKW i. A. jedes wichtige Segment der Langform von einem Segment der Kurzform vertreten wird. In den Fachsprachen ist das Kurzwort Teil des Terminus, seine Form muss also genau festgelegt werden.

(3) Drittens ist ein großer Vorteil der MKW, dass man mit ihnen leicht Komposita bilden und dadurch sehr komplexe Inhalte relativ einfach ausdrücken kann, was mit der Langform undenkbar wäre. Die Kompositionsfreudigeit der MKW ist sehr groß, in der Allgemeinsprache genauso wie in den Fachsprachen, im Deutschen genauso wie im Ungarischen. Eine ganz merkwürdige ,internationale Bildung" stellen Komposita dar, deren Glieder aus anderen Sprachen stammen (vgl. Donalies 2005:16; Murányi-Zagyvai 2017). 
(4) Ein vierter Vorteil der fachsprachlichen MKW, aber besonders der entlehnten fachsprachlichen MKW ist eben mit ihrem „Hauptnachteil“, der Unmotiviertheit verbunden. Sie bilden eine wichtige Brücke zwischen der Fachwelt und der Öffentlichkeit, indem sie Laien helfen, lange, komplizierte, (zum Teil) aus „unverständlichen“ fremdsprachlichen Elementen gebildete Langformen, die schwer auszusprechen und zu schreiben sind, durch einfache Formen zu ersetzen, die sie auch leichter lernen können (vgl. Barz 2016:749).

(5) Der fünfte Vorteil der MKW spielt in den Fachsprachen, in der internationalen Kooperation eine zentrale Rolle: MKW sind leicht zu entlehnen, ihre Assimilation ist schneller, sie wirken nicht körperfremd, und geben der Fachwelt Zeit, eine entsprechende Langform zu den schon bestehenden Kurzformen zu bilden; also MKW füllen vorübergehend die Lücken in der Terminologie der Zielsprache, wodurch sie paradoxerweise dazu beitragen, die Terminologie der Zielsprache auszubauen. $M A L D I<$ matrix-assisted laser desorption/ionization ist ein typisches Beispiel dafür, wie schwer sich ein einheimisches (ungarisches) Langform-Äquivalent gestaltet. Zur Zeit finden sich folgende Äquivalent-Varianten in Fachtexten (ohne Anspruch auf Vollständigkeit): mátrixhozkötött lézer ionizáció, mátrixhoz kötött lézer ionizáció, mátrixközvetitett lézeres deszorpció/ionizáció, mátrix által segitett lézerdeszorpciós ionizáció, mátrix segitett lézer deszorpció és ionizáció, mátrix-asszisztált lézer deszorpció/ionizáció. Solange keine Einheitlichkeit darüber besteht, welches Äquivalent terminologisiert werden soll, kann das Kurzwort die Eindeutigkeit sichern.

Die vermehrte Entlehnung der MKW hat in vielen Nationalfachsprachen (vor allem in der Fachsprache der Naturwissenschaften, der Medizin und der Technik) einen neuartigen Wandel verursacht. Dieser Wandel bezieht sich einerseits auf die Vernetzung der Nationalfachsprachen mit Hilfe eines MKW-Netzes, andererseits auf die Bereicherung des Fachwortschatzes mit einer neuen Terminologie- bzw. Nomenklatur-Basis.

Dieses Netz wird nicht nur immer größer, sondern auch ältere Formen werden neu gestaltet. Für diese neuartige Gestaltung ist ein gutes Beispiel das Buchstabenkurzwort dt. DNS $<$ Desoxyribonucleinsäure $=$ ung. $D N S<$ dezoxiribonukleinsav $=$ engl. $D N A<$ deoxyribonucleic acid. Zum englischen Kurzwort wurde zwar früher ein deutsches (und auch ein ungarisches) Äquivalent gebildet, aber das englische und das deutsche Buchstabenkurzwort werden in der letzten Zeit im Deutschen nebeneinander gebraucht, in unseren Korpustexten wird $D N A$ sogar dem deutschen Kurzwort vorgezogen, in Komposita findet man nur noch DNA. Ähnlich bei $R N S<$ Ribonucleinsäure vs. $R N A<$ ribonucleic acid. Die Tendenz ist also, dass englische MKW deutsche verdrängen, möglicherweise um der Einheitlichkeit willen. Im Ungarischen halten sich noch DNS und RNS durch. Anders ist es im Falle von EDTA < engl. ethylenediamine tetraacetic acid, dt. Ethylendiamintetraacetat, ung. etilén-diamin-tetraacetát und EDTE < dt. Ethylendiamintetraessigsäure; ung. etilén-diamin-tetraecetsav. Im Deutschen (und auch im Ungarischen) sind beide MKW (EDTA und EDTE) korrekt. Im englisch-deutschen zweisprachigen Wörterbuch der chemischen Analytik (vgl. Knepper 1995) wird als deutsches Äquivalent zum englischen MKW noch EDTE angegeben, aber in keinem deutschen (und keinem ungarischen) Korpustext wurde EDTE verwendet, EDTA dagegen oft.

Wie oben erwähnt, knüpft sich an den MKW-Gebrauch auch ein anderer Wandel, eine andere Innovation in den Fachsprachen. In der Chemie bedeutet Nomenklatur das System der Namengebung von Substanzen, die Terminologie umfasst alle anderen Fachausdrücke, z. B. Bezeichnungen von Handlungen und Vorgängen im Labor, Verfahren, Geräten und Apparaten, Gesetzen, Phänomenen, Konzepten usw. Das zuständige Organ für die chemische Nomenklatur ist der Dachverband der nationalen chemischen Gesellschaften, die IUPAC ( $<$ International Union of Pure and Applied Chemistry), die die englischen Bezeichnungen regelt. Für die nationalen Äquivalente sind die nationalen Chemikerverbände zuständig.

Zur Benennung von Verbindungen stehen in der Chemie drei Nomenklaturen zur Verfügung: die systematische Nomenklatur, die systematische Namen enthält, die Trivial-Nomenklatur, die Trivialnamen enthält und die sog. halbsystematische Nomenklatur. Systematischen Namen sind lang und kompliziert, z. B. Natriumhydrogencarbonat, haben aber den Vorteil, dass aus ihnen die chemische Struktur (Molekülstruktur) der Substanz abgeleitet werden kann. Trivialnamen sind 
kürzer und einfacher, historisch fest verwurzelt, und sie können z. B. durch verschiedene Stoffmerkmale motiviert sein, z. B. Natron. Halbsystematische Namen bilden einen Übergang zwischen systematischen und Trivialnamen, sie enthalten mindestens einen Teil eines systematischen Namens, z. B. Natriumbicarbonat. Eine relativ neue Gruppe der Bezeichnungen bilden die MKW, die aber nicht nach eindeutigen Regeln zustande gebrachte Namen, sondern eher aus der „Praxis“ kommende Ersatz-Bezeichnungen sind. Sie können nicht nur Namen von Substanzen, sondern beliebige Termini ersetzen, wenn diese in der Kommunikation ,schwerfällig“ sind. Sie sind nicht von allen Fachleuten gern gesehene Formen, es wird ihnen vorgeworfen, dass sie ,in unüberschaubarer und unkontrollierter Form überall in den Fach- und Werkstattsprachen, aber auch in Politik und Öffentlichkeit produziert werden“ (EBEL 1998:1256), aber unter den IUPAC-Bezeichnungen sind zahlreiche MKW registriert, und sind auf der Homepage der IUPAC (iupac.org) zu finden. Ohne sie sind Fachtexte heute nicht denkbar.

\section{Zusammenfassung und Ausblick}

MKW sind relativ neue, aber wichtige Elemente der Fachsprachen. In der Fachsprache der chemischen Analytik, aus der die untersuchten Beispiele in der vorliegenden Arbeit stammen, ist die Mehrheit der MKW aus dem Englischen als internationaler Wissenschaftssprache entlehnt worden, sie bezeichnet vor allem Substanzen und Verbindungen, Verfahren, Geräte oder Teile von ihnen, Messparameter sowie Maßeinheiten.

Entlehnte MKW können in den untersuchten Zielsprachen (Deutsch und Ungarisch) meist einfach gebraucht werden, weil die Langformen ihre innere morphologische Struktur bei der Kurzwortbildung verloren haben. In der fachsprachlichen Verwendung gibt es - wegen der Standardisierung - auch keinen semantischen Unterschied.

In der Schrift werden sie im Allgemeinen - ähnlich wie Eigennamen - unverändert übernommen. Das Schriftbild hat bei MKW eine besondere Bedeutung: es macht den Leser darauf aufmerksam, dass das Wort ,anders“ zu lesen ist als nicht reduzierte Formen. In den Fachtexten kann die eindeutige Bestrebung beobachtet werden, dass man die MKW durch die Majuskelschrift von den anderen Lexemen trennen will. Diese Schreibweise hat sich sowohl im Deutschen als auch im Ungarischen weitgehend durchgesetzt, auch wenn sich in den einzelnen Nationalsprachen z. T. andere orthographische Vorschriften auf MKW beziehen. Die Unterscheidung von Großbuchstaben und Kleinbuchstaben ist in den Fachsprachen nicht einfach eine orthographische Frage, die Schreibung beruht auf Vereinbarung, sie ist Teil des Fachbegriffs, eine Veränderung kann zu Missverständnissen führen.

Die Bildungsmethode der MKW, aber vor allem die der Buchstabenkurzwörter geht damit einher, dass sprachspezifische Unterschiede - in der geschriebenen Fachsprache - völlig verschwinden: englische, deutsche, ungarische MKW können an ihrem Schriftbild nicht erkannt werden, es sei denn, sie enthalten Grapheme, die in anderen Schriftsystemen nicht gebräuchlich sind. (In dieser Hinsicht ist es günstig, dass das Alphabet des Englischen, der heute aktuellen internationalen Wissenschaftssprache - im Vergleich zu anderen Sprachen, die das lateinische Alphabet benutzen keine „besonderen“ Buchstaben hat.)

MKW passen sich leicht ans System der Zielsprache an. Ihre Anpassung vollzieht sich in der Aussprache sowie in der Grammatik, in der Schreibweise kommt es praktisch zu keiner Anpassung, sie ist nicht nötig. Im Unterschied zur schriftlichen Kommunikation hat man in der mündlichen Kommunikation damit zu rechnen, dass MKW schwieriger identifiziert werden können, da sie in den Zielsprachen i. A. wie einheimische MKW ausgesprochen werden. Die grammatische Assimilation (Artikelzuweisung, Flexion) kann - je nach Zielsprache - mit mehr oder weniger Schwierigkeiten verbunden sein, aber in Notfall besteht immer die Möglichkeit der Komposition mit einem einheimischen Zweitglied.

Die Verwendung der entlehnten MKW in den untersuchten deutschen und ungarischen Texten ist unter mehreren Aspekten von Vorteil. Sie passen sich schnell an, füllen eventuelle terminologische 
Lücken in der Zielsprache, und mit der Zeit können zu ihnen entsprechende Langformen in den Nationalfachsprachen gebildet werden.

Englische und englisch transmittierte MKW stellen ein neues Wortschatzsegment, eine neue Form der Internationalismen dar, die sowohl die nationale als auch die internationale (Fach)kommunikation erleichtern können.

\section{Literaturverzeichnis}

\section{Primärliteratur:}

BAUER, Stefan (2002): Die Zusammensetzung der Oberflächenwachse von Tomaten, Paprika und Auberginen. PhD-Dissertation an der Westfälischen Wilhelms-Universität Münster.

BÉNI, Áron (2007): Mintaelőkészitési és mintabeviteli módszerek fejlesztése krómspeciációs elemzésekhez. PhD-Dissertation. Debreceni Egyetem.

Bокотеу, Sándor (2001): Bioaktív molekulák szerkezetvizsgálata korszerü NMR módszerekkel. $\mathrm{PhD}$-Dissertation. Debreceni Egyetem.

Csomós, Elemér (2003): Magyar fehér- és vörösborok összehasonlító vizsgálata a szabad aminosav és a biogén amin tartalom alapján. PhD-Dissertation. Budapesti Müszaki Egyetem.

DOMBOVÁry, János (2004): Biológiai anyagok nyomelem analitikájának fejlesztése. PhD-Dissertation. Debreceni Egyetem.

GEUKING, Holger (2007): Entwicklung eines IR-Kohlenmonoxidsensors. PhD-Dissertation an der Westfälischen Wilhelms-Universität Münster.

GYÉMÁNT, Gyöngyi (2001): A nagyhatékonyságú-folyadékkromatográfia alkalmazása szénhidrátkémiai és szénhidrát-biokémiai kutatásokban. PhD-Dissertation. Debreceni Egyetem.

HEIER, Anke (2006): Nachweis der geographischen Herkunft von Pistazien anhand der Stabilisotopenverhältnisse. PhD-Dissertation an der Technischen Universität Dresden.

JÜRGENLIEMK, Guido (2001): Phenolische Inhaltsstoffe aus dem Kraut von Hypericum perforatum L. - analytische, biopharmazeutische und pharmakologische Aspekte. PhD-Dissertation an der Westfälischen Wilhelms-Universität Münster.

KaltenBaCH, Thilo (2001): Anwendung der Kapillarelektrophorese in der Arzneimittel- und Wirkstoffprüfung. PhD-Dissertation an der Westfälischen Wilhelms-Universität Münster.

KLEMM, Denis (2008): Analyse dünner Schichten mit der optischen Glimmentladungsspektroskopie. PhD-Dissertation an der Technischen Universität Dresden.

LÁNYI, Katalin (2002): Nitrogéntartalmú herbicidek fotodegradációs folyamatainak vizsgálata gázkromatográfiás és gázkromatográfiás-tömegspektrometriás módszerrel. PhD-Dissertation. Debreceni Egyetem.

MunDRY, Tobias (1999): Einbrennsilikonisierung bei pharmazeutischen Glaspackmitteln - Analytische Studien eines Produktionsprozesses. PhD-Dissertation an der Humboldt-Universität zu Berlin.

MURÁNYI, Zoltán (2002): Újabb eredmények a borok nyomelemtartalmáról. PhD-Dissertation. Debreceni Egyetem.

RommeL, Elisabeth (2007): Tierische Zellen auf nanoporösen Oberflächen: Grundlagen und bioanalytische Anwendungen. PhD-Dissertation an der Westfälischen Wilhelms-Universität Münster.

SCHÄFFER, Richárd (2007): Csatolt technikák fejlesztése és alkalmazása arzénmódosulatok meghatározására. PhD-Dissertation an der Corvinus Egyetem. Budapest.

SCHMIDT, Torsten (2001): Identifizierung und Untersuchung pharmazeutischer Gläser durch Laser-Ablation-ICP-MS. PhD-Dissertation an der Humboldt-Universität zu Berlin.

Schwedt, Georg (2008): Analytische Chemie. Grundlagen, Methoden und Praxis. Weinheim.

SzANics, Enikő (2007): Proteomikai módszerek alkalmazása különbözö eredetü fehérjék vizsgálatára. PhD-Dissertation an der Corvinus Egyetem. Budapest.

SzILÁGYI, László (2004): Katonizáció vizsgálata MALDI körülmények között. PhD-Dissertation. Debreceni Egyetem. 
ZÁrAY, Gyula (Hrsg.) (2006): Az elemanalitika korszerü módszerei. Budapest.

ZoRn, Eva-Christina (2003): Methoden der spurenanalytischen Bestimmung von Estrogenen im Abwasser. PhD-Dissertation an der Humboldt-Universität zu Berlin.

\section{Sekundärliteratur:}

BARZ, Irmhild (2016): Die Wortbildung. In: Duden. Die Grammatik. Berlin, S. 644-774.

Bussmann, Hadumod (2002): Lexikon der Sprachwissenschaft. Stuttgart.

DonALIES, Elke (2005): Die Wortbildung des Deutschen. Ein Überblick. Tübingen.

EBEL, Hans (1998): Die neuere Fachsprache der Chemie unter besonderer Berücksichtigung der Organischen Chemie. In: HofFManN, Lothar / KALVERKÄMPER, Hartwig / Wiegand, Herbert Ernst (Hrsg.): Fachsprachen: ein internationales Handbuch zur Fachsprachenforschung und Terminologiewissenschaft. 1. Halbband. Berlin; New York, S. 1235-1259.

KNEPPER, Joachim (1995): Fachwörterbuch Chemische Analytik. Englisch-Deutsch/Deutsch-Englisch. Berlin; Paris.

Kobler-Trill, Dorothea (1994): Das Kurzwort im Deutschen. Eine Untersuchung zu Definition, Typologie und Entwicklung. Tübingen.

LACZKó, Krisztina / MárTonfi, Attila (2006): Helyesiŕ́s. Budapest.

MurÁNYI-ZAGYvaI, Márta (2014): Kurzworttypologie - ein bisschen anders. In: ŻEBRowsKa, Ewa / JaWORSKa, Mariola / STEINHOFF, Dirk (Hrsg.): Materialität und Medialität der sprachlichen Kommunikation. Akten des 47. Linguistischen Kolloquiums in Olsztyn. Frankfurt am Main; Bern; Bruxelles u. a., S. 243-253.

MurÁNYI-ZagYvaI, Márta (2017): Kurzwortkomposita als sprachlicher Schmelztiegel von Kulturen. In: FöLDES, Csaba (Hrsg.): Interkulturelle Germanistik als Forschungsorientierung in der mitteleuropäischen Germanistik. Tübingen, S. 131-147.

MuRÁNYINÉ ZAGYVAI, Márta (2017): A ritkább szóalkotási módok germanista szemmel I. A reduktív szóalkotási módok. In: ZIMÁNYI, Árpád (Hrsg.): A magyar tudomány ünnepe 2016. Tanulmányok a bölcsészettudományok köréböl. Eger, S. 93-103.

Römer, Christine / MATZKe, Brigitte (2005): Lexikologie des Deutschen. Eine Einführung. Tübingen.

SCHLAEFER, Michael (2002): Lexikologie und Lexikographie. Eine Einführung am Beispiel deutscher Wörterbücher. Berlin.

SteInHAuer, Anja (2000): Sprachökonomie durch Kurzwörter. Bildung und Verwendung in der Fachkommunikation. Tübingen. 